\title{
NON-DIFFERENTIABLE EXACT SOLUTIONS FOR THE NONLINEAR ODES DEFINED ON FRACTAL SETS
}

\author{
XIAO-JUN YANG ${ }^{*}$ FENG GAO ${ }^{*, \S}$ and H. M. SRIVASTAVA ${ }^{\dagger, \ddagger}$ \\ ${ }^{*}$ State Key Laboratory for Geo-Mechanics \\ and Deep Underground Engineering \\ School of Mechanics and Civil Engineering \\ China University of Mining and Technology \\ Xuzhou 221116, P. R. China \\ ${ }^{\dagger}$ Department of Mathematics and Statistics \\ University of Victoria, Victoria, British Columbia V8W 3R4, Canada \\ ${ }^{\ddagger}$ Department of Medical Research, China Medical University Hospital \\ China Medical University, Taichung 40402, Taiwan, Republic of China \\ §jsppw@sohu.com
}

Received February 23, 2017

Revised April 5, 2017

Accepted April 10, 2017

Published July 6, 2017

\begin{abstract}
In the present paper, a family of the special functions via the celebrated Mittag-Leffler function defined on the Cantor sets is investigated. The nonlinear local fractional ODEs (NLFODEs) are presented by following the rules of local fractional derivative (LFD). The exact solutions for these problems are also discussed with the aid of the non-differentiable charts on Cantor sets. The obtained results are important for describing the characteristics of the fractal special functions.
\end{abstract}

Keywords: Nonlinear ODEs; Local Fractional Derivative; Mittag-Leffler Function; Cantor Sets.

\footnotetext{
$\S$ Corresponding author.

This is an Open Access article published by World Scientific Publishing Company. It is distributed under the terms of the Creative Commons Attribution 4.0 (CC-BY) License. Further distribution of this work is permitted, provided the original work is properly cited.
} 


\section{INTRODUCTION}

Fractional ordinary differential equations (FODEs $)^{1-3}$ have been successfully used to model the complexity in mathematics, physics and societies, such as the fractional evolution, ${ }^{4}$ control, ${ }^{5}$ circuits,$~^{6-9}$ relaxation $^{10-14}$ and population dynamics. ${ }^{15}$ Finding the solutions for these mentioned models, many technologies were proposed in Ref. 16. For example, the Adomian decomposition technology (ADT) ${ }^{17}$ and its extended ver$\operatorname{sion}^{18}$ were proposed to solve the approximate solutions for the FODEs. The spectral element method $(\mathrm{SEM})^{19}$ and the finite difference method (FDM $)^{20}$ and the linear multiple step method (LMSM) ${ }^{21}$ were discussed to handle the numerical solutions for the FODEs. The technologies involving the differential transform (DT) ${ }^{22}$ and the fractional operational calculus (FOC) ${ }^{23}$ technologies were reported in order to find the analytical and exact solutions for FODEs, respectively.

Recently, fractional calculus (FC) was considered to solve a class of the fractal problems in mathematical physics, ${ }^{24-28}$ mechanics, ${ }^{29-31}$ heat,${ }^{32}$ biology ${ }^{33}$ and others. ${ }^{34-37}$ There is an alternative operator (called local FC) to model the local FODEs in fractal electric circuits, ${ }^{38}$ free damped vibrations, ${ }^{39}$ shallow water surfaces ${ }^{40}$ and populations. ${ }^{41-43}$ The fractal partial differential equations (FPDEs) in mathematical physics were also discussed in Refs. 44-48. The structure solutions for the nonlinear local fractional ordinary differential equations (NLFODEs) have not been sufficiently investigated. Motivated especially by the above idea, our aim in the present article is to structure the NLFODEs by means of a family of the special functions via the Mittag-Leffler function defined on the Cantor sets.

The structure of the paper is designed as follows. In Sec. 2, the basic definitions of the local fractional derivative (LFD) and special functions defined on Cantor sets are introduced. In Sec. 3, we present the NLFODEs with the use of the LFDs of the special functions defined on the Cantor sets. Finally, we give the conclusion in Sec. 4 .

\section{PRELIMINARIES, DEFINITIONS AND FRACTAL SPECIAL FUNCTIONS}

Definition 1. The LFD of $\Pi_{\tau}(\mu)$ of fractal order $\tau(0<\tau<1)$ at the point $\mu=\mu_{0}$ is defined by $24,38-41,44-48$

$$
\begin{aligned}
D^{(\tau)} & \Pi_{\tau}\left(\mu_{0}\right) \\
& =\frac{d^{\tau} \Pi_{\tau}\left(\mu_{0}\right)}{d \mu^{\tau}} \\
& =\lim _{\mu \rightarrow \mu_{0}} \frac{\Delta^{\tau}\left(\Pi_{\tau}(\mu)-\Pi_{\tau}\left(\mu_{0}\right)\right)}{\left(\mu-\mu_{0}\right)^{\tau}},
\end{aligned}
$$

where

$$
\begin{aligned}
& \Delta^{\tau}\left(\Pi_{\tau}(\mu)-\Pi_{\tau}\left(\mu_{0}\right)\right) \\
& \quad \cong \Gamma(1+\tau) \Delta\left[\Pi_{\tau}(\mu)-\Pi_{\tau}\left(\mu_{0}\right)\right] .
\end{aligned}
$$

Definition 2. The LFD of $\Pi_{\tau}(\mu)$ of fractal order $\kappa \tau(0<\tau<1, \kappa \in \mathrm{N})$ at the point $\mu=\mu_{0}$ is given as follows (see Refs. 24 and 43):

$$
D^{(\kappa \tau)} \Pi_{\tau}\left(\mu_{0}\right)=\underbrace{\frac{d^{\tau}}{d \mu^{\tau}} \cdots \frac{\partial^{\tau}}{\partial \mu^{\tau}}}_{\kappa \text { times }} \Pi_{\tau}\left(\mu_{0}\right) .
$$

If $j^{\tau}$ is a fractal imaginary unit and $\kappa \in \mathbb{N}_{0}$, then the fractal special functions defined on fractal sets ${ }^{24,38-41,43-48}$ are listed in Table 1, $\mathbb{N}_{0}$ being (as usual) the set of nonnegative integers.

If $\rho$ is a constant, then the LFDs of the fractal special functions defined on fractal sets ${ }^{24,38-41,44-48}$ are listed in Table 2.

\section{NONLINEAR LOCAL FRACTIONAL ODES}

In this section, we apply the results of the LFDs of the special functions defined on Cantor sets in order to structure the NLFODEs.

Defining the following special functions on Cantor sets:

$$
\Phi_{\tau}(\mu)=\varphi_{1} \sin _{\tau}\left(\varphi_{2} \mu^{\tau}\right)
$$

and

$$
\Phi_{\tau}(\mu)=\varphi_{1} \cos _{\tau}\left(\varphi_{2} \mu^{\tau}\right),
$$

where $\varphi_{1}$ and $\varphi_{2}$ are two parameters, we find from Table 2 that

$$
D^{(\tau)} \varphi_{1} \sin _{\tau}\left(\varphi_{2} \mu^{\tau}\right)=\varphi_{1} \varphi_{2} \cos _{\tau}\left(\varphi_{2} \mu^{\tau}\right)
$$

and

$$
D^{(\tau)} \varphi_{1} \cos _{\tau}\left(\varphi_{2} \mu^{\tau}\right)=-\varphi_{1} \varphi_{2} \sin _{\tau}\left(\varphi_{2} \mu^{\tau}\right),
$$

so that we get the following NLFODE:

$$
\left[D^{(\tau)} \Phi_{\tau}(\mu)\right]^{2}=\varphi_{2}^{2}\left(\varphi_{1}^{2}-\Phi_{\tau}^{2}(\mu)\right) .
$$

When $\varphi_{1}=1$ and $\varphi_{2}=1$, from Eq. (8), we get the NLFODE as follows:

$$
\left[D^{(\tau)} \Phi_{\tau}(\mu)\right]^{2}=1-\Phi_{\tau}^{2}(\mu)
$$


Table 1 The Expressions of the Fractal Special Functions.

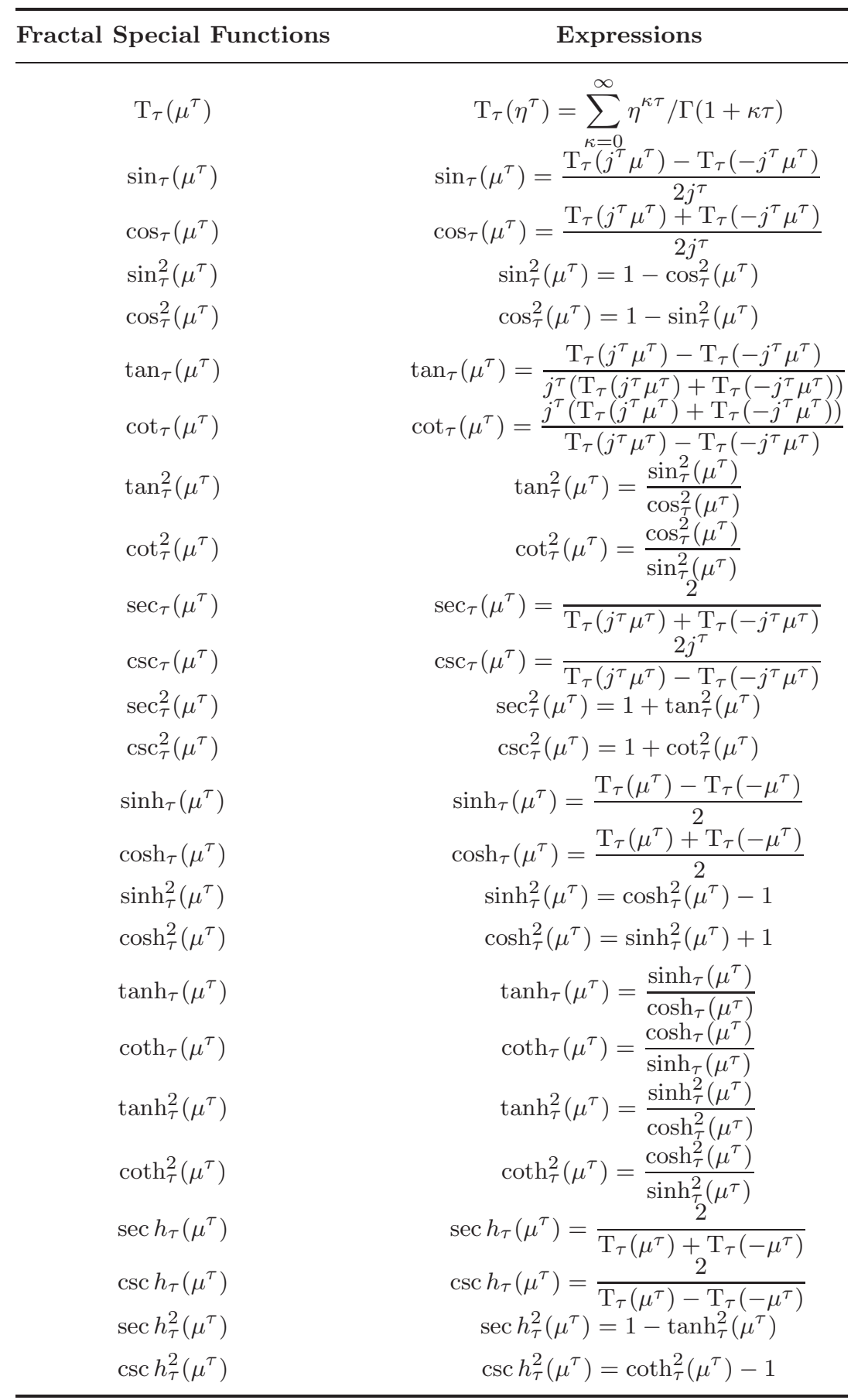

where the non-differentiable solution has the form given by

$$
\Phi_{\tau}(\mu)=\left\{\begin{array}{l}
\varphi_{1} \sin _{\tau}\left(\varphi_{2} \mu^{\tau}\right) \\
\varphi_{1} \cos _{\tau}\left(\varphi_{2} \mu^{\tau}\right)
\end{array}\right.
$$

Similarly, by taking the following special functions defined on Cantor sets:

$$
\Phi_{\tau}(\mu)=\varphi_{1} \sinh _{\tau}\left(\varphi_{2} \mu^{\tau}\right)
$$

and

$$
\Phi_{\tau}(\mu)=\varphi_{1} \cosh _{\tau}\left(\varphi_{2} \mu^{\tau}\right)
$$

we have

$$
D^{(\tau)} \varphi_{1} \sinh _{\tau}\left(\varphi_{2} \mu^{\tau}\right)=\varphi_{1} \varphi_{2} \cosh _{\tau}\left(\varphi_{2} \mu^{\tau}\right)
$$

and

$$
D^{(\tau)} \varphi_{1} \cosh _{\tau}\left(\varphi_{2} \mu^{\tau}\right)=\varphi_{1} \varphi_{2} \sinh _{\tau}\left(\varphi_{2} \mu^{\tau}\right)
$$


Table 2 The LFDs of the Fractal Special Functions Defined on Fractal Sets.

\begin{tabular}{cc}
\hline Fractal Special Functions & LFDs \\
\hline $\mathrm{T}_{\tau}\left(\rho \mu^{\tau}\right)$ & $D^{(\tau)} \mathrm{T}_{\tau}\left(\rho \mu^{\tau}\right)=\rho \mathrm{T}_{\tau}\left(\rho \mu^{\tau}\right)$ \\
$\sin _{\tau}\left(\rho \mu^{\tau}\right)$ & $D^{(\tau)} \sin _{\tau}\left(\rho \mu^{\tau}\right)=\rho \cos _{\tau}\left(\rho \mu^{\tau}\right)$ \\
$\cos _{\tau}\left(\rho \mu^{\tau}\right)$ & $D^{(\tau)} \cos _{\tau}\left(\rho \mu^{\tau}\right)=-\rho \sin _{\tau}\left(\rho \mu^{\tau}\right)$ \\
$\tan _{\tau}\left(\rho \mu^{\tau}\right)$ & $D^{(\tau)} \tan _{\tau}\left(\rho \mu^{\tau}\right)=\rho\left(1+\tan _{\tau}^{2}\left(\rho \mu^{\tau}\right)\right)$ \\
$\cot _{\delta}\left(\rho \tau^{\delta}\right)$ & $D^{(\tau)} \cot _{\tau}\left(\rho \mu^{\tau}\right)=-\rho\left(1+\cot _{\tau}^{2}\left(\rho \mu^{\tau}\right)\right)$ \\
$\sec _{\tau}\left(\rho \mu^{\tau}\right)$ & $D^{(\tau)} \sec _{\tau}\left(\rho \mu^{\tau}\right)=\rho \sec _{\tau}\left(\rho \mu^{\tau}\right) \tan _{\tau}\left(\rho \mu^{\tau}\right)$ \\
$\csc _{\tau}\left(\rho \mu^{\tau}\right)$ & $D^{(\tau)} \csc _{\tau}\left(\rho \mu^{\tau}\right)=-\rho \csc _{\tau}\left(\rho \mu^{\tau}\right) \cot _{\tau}\left(\rho \mu^{\tau}\right)$ \\
$\sinh _{\tau}\left(\rho \mu^{\tau}\right)$ & $D^{(\tau)} \sinh _{\tau}\left(\rho \mu^{\tau}\right)=\rho \cosh _{\tau}\left(\rho \mu^{\tau}\right)$ \\
$\cosh _{\tau}\left(\rho \mu^{\tau}\right)$ & $D^{(\tau)} \cosh _{\delta}\left(\rho \tau^{\delta}\right)=\rho \sinh _{\delta}\left(\rho \tau^{\delta}\right)$ \\
$\tanh _{\tau}\left(\rho \mu^{\tau}\right)$ & $D^{(\tau)} \tanh _{\tau}\left(\rho \mu^{\tau}\right)=\rho\left(1-\tanh _{\delta}^{2}\left(\rho \tau^{\delta}\right)\right)$ \\
$\operatorname{coth}_{\tau}\left(\rho \mu^{\tau}\right)$ & $D^{(\tau)} \operatorname{coth}_{\tau}\left(\rho \mu^{\tau}\right)=-\rho\left(1-\operatorname{coth}_{\tau}^{2}\left(\rho \mu^{\tau}\right)\right)$ \\
$\sec _{\tau}\left(\rho \mu^{\tau}\right)$ & $D^{(\tau)} \sec _{\tau}\left(\rho \mu^{\tau}\right)=-\rho \sec h_{\tau}\left(\rho \mu^{\tau}\right) \tanh _{\tau}\left(\rho \mu^{\tau}\right)$ \\
$\csc _{\tau}\left(\rho \mu^{\tau}\right)$ & $D^{(\tau)} \csc _{\tau}\left(\rho \mu^{\tau}\right)=-\rho \csc h_{\tau}\left(\rho \mu^{\tau}\right) \operatorname{coth}_{\tau}\left(\rho \mu^{\tau}\right)$ \\
\hline
\end{tabular}

so that we present the form of the NLFODE as follows:

$$
\left[D^{(\tau)} \Phi_{\tau}(\mu)\right]^{2}=\varphi_{2}^{2}\left(\Phi_{\tau}^{2}(\mu)-\varphi_{1}^{2}\right) .
$$

Thus, we easily structure from Eqs. (8) and (15), the following NLFODE:

$$
\left[D^{(\tau)} \Phi_{\tau}(\mu)\right]^{2}=\nu \varphi_{2}^{2}\left(\Phi_{\tau}^{2}(\mu)-\varphi_{1}^{2}\right),
$$

where the non-differentiable solutions can be written as follows:

$$
\Phi_{\tau}(\mu)= \begin{cases}\varphi_{1} \sin _{\tau}\left(\varphi_{2} \mu^{\tau}\right), & (\nu=-1), \\ \varphi_{1} \cos _{\tau}\left(\varphi_{2} \mu^{\tau}\right), & (\nu=-1), \\ \varphi_{1} \sinh _{\tau}\left(\varphi_{2} \mu^{\tau}\right), & (\nu=1), \\ \varphi_{1} \cosh _{\tau}\left(\varphi_{2} \mu^{\tau}\right), & (\nu=1) .\end{cases}
$$

In a similar manner, we consider the following special functions defined on Cantor sets:

$$
\Phi_{\tau}(\mu)=\varphi_{1} \tan _{\tau}\left(\varphi_{1} \mu^{\tau}\right)
$$

and

$$
\Phi_{\tau}(\mu)=\varphi_{1} \cot _{\tau}\left(\varphi_{1} \mu^{\tau}\right) .
$$

In view of Eqs. (18) and (19), we have

$$
D^{(\tau)} \varphi_{1} \tan _{\tau}\left(\varphi_{1} \mu^{\tau}\right)=\varphi_{1} \varphi_{2}\left(1+\tan _{\tau}^{2}\left(\varphi_{2} \mu^{\tau}\right)\right)
$$

and

$$
D^{(\tau)} \varphi_{1} \cot _{\tau}\left(\varphi_{2} \mu^{\tau}\right)=-\varphi_{1} \varphi_{2}\left(1+\cot _{\tau}^{2}\left(\varphi_{2} \mu^{\tau}\right)\right)
$$

so that

$$
D^{(\tau)} \Phi_{\tau}(\mu)= \pm \varphi_{2}\left(\varphi_{1}+\frac{1}{\varphi_{1}} \Phi_{\tau}^{2}(\mu)\right) .
$$

Thus, we directly obtain the following NLFODE:

$$
D^{(\tau)} \Phi_{\tau}(\mu)=\nu \varphi_{2}\left(\varphi_{1}+\frac{1}{\varphi_{1}} \Phi_{\tau}^{2}(\mu)\right),
$$

where $\nu$ is a parameter and the non-differentiable solutions can be given as follows:

$$
\Phi_{\tau}(\mu)= \begin{cases}\varphi_{1} \tan _{\tau}\left(\varphi_{2} \mu^{\tau}\right), & (\nu=1), \\ \varphi_{1} \cot _{\tau}\left(\varphi_{2} \mu^{\tau}\right), & (\nu=-1) .\end{cases}
$$

In a similar manner, we can structure the following NLFODE:

$$
D^{(\tau)} \Phi_{\tau}(\mu)=\varphi_{2}\left(\varphi_{1}-\frac{1}{\varphi_{1}} \Phi_{\tau}^{2}(\mu)\right),
$$

where the non-differentiable solution is represented by

$$
\Phi_{\tau}(\mu)=\left\{\begin{array}{l}
\varphi_{1} \tanh _{\tau}\left(\varphi_{2} \mu^{\tau}\right), \\
\varphi_{1} \operatorname{coth}_{\tau}\left(\varphi_{2} \mu^{\tau}\right)
\end{array}\right.
$$

Making use of Eqs. (23) and (25), we can derive the following NLFODE:

$$
D^{(\tau)} \Phi_{\tau}(\mu)= \pm \varphi_{2}\left(\varphi_{1}+\frac{\nu}{\varphi_{1}} \Phi_{\tau}^{2}(\mu)\right),
$$

where the non-differentiable solutions are given by

$$
\Phi_{\tau}(\mu)= \begin{cases}\varphi_{1} \sinh _{\tau}\left(\varphi_{2} \mu^{\tau}\right), & (\nu=1), \\ \varphi_{1} \cosh _{\tau}\left(\varphi_{2} \mu^{\tau}\right), & (\nu=1), \\ \varphi_{1} \tanh _{\tau}\left(\varphi_{2} \mu^{\tau}\right), & (\nu=-1), \\ \varphi_{1} \operatorname{coth}_{\tau}\left(\varphi_{2} \mu^{\tau}\right), & (\nu=-1) .\end{cases}
$$

Let us define the following special functions on Cantor sets:

$$
\begin{aligned}
& \Phi_{\tau}(\mu)=\varphi_{1} \sec _{\tau}\left(\varphi_{2} \mu^{\tau}\right), \\
& \Phi_{\tau}(\mu)=\varphi_{1} \csc _{\tau}\left(\varphi_{2} \mu^{\tau}\right), \\
& \Phi_{\tau}(\mu)=\varphi_{1} \sec h_{\tau}\left(\varphi_{2} \mu^{\tau}\right)
\end{aligned}
$$


and

$$
\Phi_{\tau}(\mu)=\varphi_{1} \csc h_{\tau}\left(\varphi_{2} \mu^{\tau}\right) .
$$

From Eqs. (29)-(32), we can establish the following formulas:

$$
\begin{aligned}
& D^{(\tau)} \varphi_{1} \sec _{\tau}\left(\varphi_{2} \mu^{\tau}\right) \\
& \quad=\varphi_{1} \varphi_{2} \sec _{\tau}\left(\varphi_{2} \mu^{\tau}\right) \tan _{\tau}\left(\varphi_{2} \mu^{\tau}\right), \\
& D^{(\tau)} \varphi_{1} \csc _{\tau}\left(\varphi_{2} \mu^{\tau}\right) \\
& \quad=\varphi_{1} \varphi_{2} \csc _{\tau}\left(\varphi_{2} \mu^{\tau}\right) \cot _{\tau}\left(\varphi_{2} \mu^{\tau}\right), \\
& D^{(\tau)} \varphi_{1} \sec h_{\tau}\left(\varphi_{2} \mu^{\tau}\right) \\
& \quad=-\varphi_{1} \varphi_{2} \sec h_{\tau}\left(\varphi_{2} \mu^{\tau}\right) \tanh _{\tau}\left(\varphi_{2} \mu^{\tau}\right)
\end{aligned}
$$

and

$$
\begin{aligned}
& D^{(\tau)} \varphi_{1} \csc h_{\tau}\left(\varphi_{2} \mu^{\tau}\right) \\
& \quad=-\varphi_{1} \varphi_{2} \csc h_{\tau}\left(\varphi_{2} \mu^{\tau}\right) \operatorname{coth}_{\tau}\left(\varphi_{2} \mu^{\tau}\right) .
\end{aligned}
$$

From Eqs. (33)-(36), we have

$$
\begin{aligned}
& {\left[D^{(\tau)} \varphi_{1} \sec _{\tau}\left(\varphi_{2} \mu^{\tau}\right)\right]^{2}} \\
& \quad=\varphi_{2}^{2} \sec _{\tau}^{2}\left(\varphi_{2} \mu^{\tau}\right)\left(\varphi_{1}^{2} \sec _{\tau}^{2}\left(\varphi_{2} \mu^{\tau}\right)-\varphi_{1}^{2}\right), \\
& {\left[D^{(\tau)} \varphi_{1} \csc _{\tau}\left(\varphi_{2} \mu^{\tau}\right)\right]^{2}} \\
& \quad=\varphi_{2}^{2} \csc _{\tau}^{2}\left(\varphi_{2} \mu^{\tau}\right)\left(\varphi_{1}^{2} \cot _{\tau}^{2}\left(\varphi_{2} \mu^{\tau}\right)-\varphi_{1}^{2}\right), \\
& {\left[D^{(\tau)} \varphi_{1} \sec h_{\tau}\left(\varphi_{2} \mu^{\tau}\right)\right]^{2}} \\
& \quad=\varphi_{2}^{2} \sec h_{\tau}^{2}\left(\varphi_{2} \mu^{\tau}\right)\left[\varphi_{1}^{2}-\varphi_{1}^{2} \sec h_{\tau}^{2}\left(\varphi_{2} \mu^{\tau}\right)\right]
\end{aligned}
$$

and

$$
\begin{aligned}
& {\left[D^{(\tau)} \varphi_{1} \csc h_{\tau}\left(\varphi_{2} \mu^{\tau}\right)\right]^{2}} \\
& \quad=\varphi_{2}^{2} \csc h_{\tau}^{2}\left(\varphi_{2} \mu^{\tau}\right)\left[\varphi_{1}^{2} \csc h_{\tau}^{2}\left(\varphi_{2} \mu^{\tau}\right)+\varphi_{1}^{2}\right],
\end{aligned}
$$

so that

$$
\begin{aligned}
& {\left[D^{(\tau)} \Phi_{\tau}(\mu)\right]^{2}} \\
& \quad=\frac{\nu_{1} \varphi_{2}^{2}}{\varphi_{1}^{2}} \Phi_{\tau}^{2}(\mu)\left[\Phi_{\tau}^{2}(\mu)+\nu_{2} \varphi_{1}^{2}\right],
\end{aligned}
$$

where the non-differentiable solutions are given as follows:

$$
\begin{aligned}
& \Phi_{\tau}(\mu) \\
& \quad= \begin{cases}\varphi_{1} \sec _{\tau}\left(\varphi_{2} \mu^{\tau}\right), & \left(\nu_{1}=1 ; \nu_{2}=-1\right), \\
\varphi_{1} \csc _{\tau}\left(\varphi_{2} \mu^{\tau}\right), & \left(\nu_{1}=1 ; \nu_{2}=-1\right), \\
\varphi_{1} \sec h_{\tau}\left(\varphi_{2} \mu^{\tau}\right), & \left(\nu=-1 ; \nu_{2}=-1\right), \\
\varphi_{1} \csc h_{\tau}\left(\varphi_{2} \mu^{\tau}\right), & \left(\nu=1 ; \nu_{2}=1\right) .\end{cases}
\end{aligned}
$$

From Table 1, we set up the following special function defined on Cantor sets:

$$
\Phi_{\tau}(\mu)=\frac{\varphi_{3}}{\left[\varphi_{1}-\varphi_{2} \mathrm{~T}_{\tau}\left(\rho \mu^{\tau}\right)\right]^{\frac{2}{\kappa}}},
$$

where $\rho, \varphi_{1}, \varphi_{2}$ and $\varphi_{3}$ are parameters.

From Eq. (43), we easily have

$$
\mathrm{T}_{\tau}\left(\rho \mu^{\tau}\right)=\frac{\varphi_{1}-\frac{2}{\kappa} \sqrt{\frac{\varphi_{3}}{\Phi_{\tau}(\mu)}}}{\varphi_{2}}
$$

and

$$
\sqrt[\frac{2}{\kappa}]{\frac{\Phi_{\tau}(\mu)}{\varphi_{3}}}=\frac{1}{\varphi_{1}-\varphi_{2} \mathrm{~T}_{\tau}\left(\rho \mu^{\tau}\right)} .
$$

For finding the LFD of Eq. (43), we present

$$
D^{(\tau)} \Phi_{\tau}(\mu)=\frac{\frac{2 \varphi_{3} \varphi_{2} \rho}{\kappa} \mathrm{T}_{\tau}\left(\rho \mu^{\tau}\right)}{\left(\varphi_{1}-\varphi_{2} \mathrm{~T}_{\tau}\left(\rho \mu^{\tau}\right)\right)^{\frac{2}{\kappa}+1}} .
$$

Upon substituting Eqs. (44) and (45) into Eq. (46), we have

$$
D^{(\tau)} \Phi_{\tau}(\mu)=\frac{2 \rho \Phi_{\tau}(\mu)}{\kappa}\left(\varphi_{1}\left(\frac{\Phi_{\tau}(\mu)}{\varphi_{3}}\right)^{\frac{\kappa}{2}}-1\right) .
$$

When $\kappa=1$, we find from Eq. (47) that

$$
D^{(\tau)} \Phi_{\tau}(\mu)=\frac{2 \rho \varphi_{1}}{\kappa \varphi_{3}} \Phi_{\tau}^{2}(\mu)-\frac{2 \rho}{\kappa} \Phi_{\tau}(\mu)
$$

together with the non-differentiable solution given by

$$
\Phi_{\tau}(\mu)=\frac{\varphi_{3}}{\varphi_{1}-\varphi_{2} \mathrm{~T}_{\tau}\left(\rho \mu^{\tau}\right)} .
$$

Similarly, we propose the following special function defined on Cantor sets:

$$
\Phi_{\tau}(\mu)=\frac{\varphi_{3}}{\left[\varphi_{1}-\varphi_{2} \mathrm{~T}_{\tau}\left(-\rho \mu^{\tau}\right)\right]^{\frac{2}{\kappa}}},
$$

which leads to

$$
\mathrm{T}_{\tau}\left(-\rho \mu^{\tau}\right)=\frac{\varphi_{1}-\frac{2}{\kappa} \sqrt{\frac{\varphi_{3}}{\Phi_{\tau}(\mu)}}}{\varphi_{2}}
$$

and

$$
\sqrt[\frac{2}{\kappa}]{\frac{\Phi_{\tau}(\mu)}{\varphi_{3}}}=\frac{1}{\varphi_{1}-\varphi_{2} \mathrm{~T}_{\tau}\left(-\rho \mu^{\tau}\right)} .
$$

In order to find the LFD of Eq. (49), we have

$$
D^{(\tau)} \Phi_{\tau}(\mu)=\frac{-\frac{2 \varphi_{3} \varphi_{2} \rho}{\kappa} \mathrm{T}_{\tau}\left(-\rho \mu^{\tau}\right)}{\left(\varphi_{1}-\varphi_{2} \mathrm{~T}_{\tau}\left(-\rho \mu^{\tau}\right)\right)^{\frac{2}{\kappa}+1}},
$$


which leads to

$$
\begin{aligned}
D^{(\tau)} & \Phi_{\tau}(\mu) \\
& =\frac{2 \rho \Phi_{\tau}(\mu)}{\kappa}\left(1-\varphi_{1}\left(\frac{\Phi_{\tau}(\mu)}{\varphi_{3}}\right)^{\frac{\kappa}{2}}\right),
\end{aligned}
$$

where $\rho, \varphi_{1}, \varphi_{2}$ and $\varphi_{3}$ are parameters.

When $\kappa=\varphi_{1}=1$ and $\varphi_{3}=1$, we find from Eq. (54) that (see Ref. 41)

$$
D^{(\tau)} \Phi_{\tau}(\mu)=2 \rho \Phi_{\tau}(\mu)\left(1-\Phi_{\tau}(\mu)\right),
$$

where the non-differentiable solution becomes (see Ref. 41)

$$
\Phi_{\tau}(\mu)=\frac{1}{1-\varphi_{2} \mathrm{~T}_{\tau}\left(-\rho \mu^{\tau}\right)}
$$

with the parameters $\rho$ and $\varphi_{2}$.

Let us now consider the following special functions defined on Cantor sets:

$$
\Phi_{\tau}(\mu)=\varphi_{1} \sin _{\tau}^{2}\left(\varphi_{2} \mu^{\tau}\right)
$$

and

$$
\Phi_{\tau}(\mu)=\varphi_{1} \cos _{\tau}^{2}\left(\varphi_{2} \mu^{\tau}\right) .
$$

By finding the LFDs of Eqs. (57) and (58), we have

$$
\begin{aligned}
& D^{(\tau)} \varphi_{1} \sin _{\tau}^{2}\left(\varphi_{2} \mu^{\tau}\right) \\
& \quad=2 \varphi_{1} \varphi_{2} \sin _{\tau}\left(\varphi_{2} \mu^{\tau}\right) \cos _{\tau}\left(\varphi_{2} \mu^{\tau}\right)
\end{aligned}
$$

and

$$
\begin{aligned}
& D^{(\tau)} \varphi_{1} \cos _{\tau}^{2}\left(\varphi_{2} \mu^{\tau}\right) \\
& \quad=-2 \varphi_{1} \varphi_{2} \cos _{\tau}\left(\varphi_{2} \mu^{\tau}\right) \sin _{\tau}\left(\varphi_{2} \mu^{\tau}\right),
\end{aligned}
$$

which yield

$$
\begin{aligned}
& {\left[D^{(\tau)} \varphi_{1} \sin _{\tau}^{2}\left(\varphi_{2} \mu^{\tau}\right)\right]^{2}} \\
& \quad=\left(2 \varphi_{1} \varphi_{2}\right)^{2} \sin _{\tau}^{2}\left(\varphi_{2} \mu^{\tau}\right) \cos _{\tau}^{2}\left(\varphi_{2} \mu^{\tau}\right)
\end{aligned}
$$

and

$$
\begin{aligned}
& {\left[D^{(\tau)} \varphi_{1} \cos _{\tau}^{2}\left(\varphi_{2} \mu^{\tau}\right)\right]^{2}} \\
& \quad=\left(2 \varphi_{1} \varphi_{2}\right)^{2} \cos _{\tau}^{2}\left(\varphi_{2} \mu^{\tau}\right) \sin _{\tau}^{2}\left(\varphi_{2} \mu^{\tau}\right) .
\end{aligned}
$$

From Eqs. (61) and (62), we get

$$
\begin{aligned}
& {\left[D^{(\tau)} \varphi_{1} \sin _{\tau}^{2}\left(\varphi_{2} \mu^{\tau}\right)\right]^{2}} \\
& \quad=\left(2 \varphi_{1} \varphi_{2}\right)^{2} \sin _{\tau}^{2}\left(\varphi_{2} \mu^{\tau}\right)\left(1-\sin _{\tau}^{2}\left(\varphi_{2} \mu^{\tau}\right)\right)
\end{aligned}
$$

and

$$
\begin{aligned}
& {\left[D^{(\tau)} \varphi_{1} \cos _{\tau}^{2}\left(\varphi_{2} \mu^{\tau}\right)\right]^{2}} \\
& \quad=\left(2 \varphi_{1} \varphi_{2}\right)^{2} \cos _{\tau}^{2}\left(\varphi_{2} \mu^{\tau}\right)\left(1-\cos _{\tau}^{2}\left(\varphi_{2} \mu^{\tau}\right)\right)
\end{aligned}
$$

which lead us to the following NLFODE:

$$
\left[D^{(\tau)} \Phi_{\tau}(\mu)\right]^{2}=\left(2 \varphi_{1} \varphi_{2}\right)^{2} \frac{\Phi_{\tau}(\mu)}{\varphi_{1}}\left(1-\frac{\Phi_{\tau}(\mu)}{\varphi_{1}}\right) .
$$

Thus, from Eq. (65), we easily obtain the following NLFODE:

$$
\left[D^{(\tau)} \Phi_{\tau}(\mu)\right]^{2}=4 \varphi_{2}^{2}\left(\varphi_{1} \Phi_{\tau}(\mu)-\Phi_{\tau}^{2}(\mu)\right),
$$

where the non-differentiable solutions are presented as follows:

$$
\Phi_{\tau}(\mu)=\left\{\begin{array}{l}
\varphi_{1} \sin _{\tau}^{2}\left(\varphi_{2} \mu^{\tau}\right), \\
\varphi_{1} \cos _{\tau}^{2}\left(\varphi_{2} \mu^{\tau}\right) .
\end{array}\right.
$$

By a similar process, we present the special functions defined on Cantor sets as follows:

$$
\Phi_{\tau}(\mu)=\varphi_{1} \sinh _{\tau}^{2}\left(\varphi_{2} \mu^{\tau}\right)
$$

and

$$
\Phi_{\tau}(\mu)=\varphi_{1} \cosh _{\tau}^{2}\left(\varphi_{2} \mu^{\tau}\right),
$$

which lead us to the following formulas:

$$
\begin{aligned}
& D^{(\tau)} \varphi_{1} \sinh _{\tau}^{2}\left(\varphi_{2} \mu^{\tau}\right) \\
& \quad=2 \varphi_{1} \varphi_{2} \sinh _{\tau}\left(\varphi_{2} \mu^{\tau}\right) \cosh _{\tau}\left(\varphi_{2} \mu^{\tau}\right)
\end{aligned}
$$

and

$$
\begin{aligned}
& D^{(\tau)} \varphi_{1} \cosh _{\tau}^{2}\left(\varphi_{2} \mu^{\tau}\right) \\
& \quad=-2 \varphi_{1} \varphi_{2} \cosh _{\tau}\left(\varphi_{2} \mu^{\tau}\right) \sinh _{\tau}\left(\varphi_{2} \mu^{\tau}\right),
\end{aligned}
$$

respectively. Therefore, we find from Eqs. (70) and (71) that

$$
\begin{aligned}
& {\left[D^{(\tau)} \varphi_{1} \sinh _{\tau}^{2}\left(\varphi_{2} \mu^{\tau}\right)\right]^{2}} \\
& \quad=\left(2 \varphi_{1} \varphi_{2}\right)^{2} \sinh _{\tau}^{2}\left(\varphi_{2} \mu^{\tau}\right)\left(1+\sinh _{\tau}^{2}\left(\varphi_{2} \mu^{\tau}\right)\right)
\end{aligned}
$$

and

$$
\begin{aligned}
& {\left[D^{(\tau)} \varphi_{1} \cosh _{\tau}^{2}\left(\varphi_{2} \mu^{\tau}\right)\right]^{2}} \\
& \quad=\left(2 \varphi_{1} \varphi_{2}\right)^{2} \cosh _{\tau}^{2}\left(\varphi_{2} \mu^{\tau}\right)\left(\cosh _{\tau}^{2}\left(\varphi_{2} \mu^{\tau}\right)-1\right),
\end{aligned}
$$

which deduce to

$$
\begin{aligned}
& {\left[D^{(\tau)} \Phi_{\tau}(\mu)\right]^{2}} \\
& \quad=\left(2 \varphi_{1} \varphi_{2}\right)^{2} \frac{\Phi_{\tau}(\mu)}{\varphi_{1}}\left(1+\frac{\Phi_{\tau}(\mu)}{\varphi_{1}}\right)
\end{aligned}
$$

and

$$
\begin{aligned}
& {\left[D^{(\tau)} \Phi_{\tau}(\mu)\right]^{2}} \\
& \quad=\left(2 \varphi_{1} \varphi_{2}\right)^{2} \frac{\Phi_{\tau}(\mu)}{\varphi_{1}}\left(\frac{\Phi_{\tau}(\mu)}{\varphi_{1}}-1\right),
\end{aligned}
$$

respectively. 
Next, from Eqs. (74) and (75), there exists the following NLFODE:

$$
\left[D^{(\tau)} \Phi_{\tau}(\mu)\right]^{2}=4 \varphi_{2}^{2} \Phi_{\tau}(\mu)\left(\Phi_{\tau}(\mu)+\nu \varphi_{1}^{2}\right),
$$

where the non-differentiable solutions are given by

$$
\Phi_{\tau}(\mu)= \begin{cases}\varphi_{1} \sinh _{\tau}^{2}\left(\varphi_{2} \mu^{\tau}\right), & (\nu=1), \\ \varphi_{1} \cosh _{\tau}^{2}\left(\varphi_{2} \mu^{\tau}\right), & (\nu=-1) .\end{cases}
$$

If the special functions defined on Cantor sets are given as follows:

$$
\Phi_{\tau}(\mu)=\varphi_{1} \tan _{\tau}^{2}\left(\varphi_{2} \mu^{\tau}\right)
$$

and

$$
\Phi_{\tau}(\mu)=\varphi_{1} \cot _{\tau}^{2}\left(\varphi_{2} \mu^{\tau}\right),
$$

then we have

$$
\begin{aligned}
& D^{(\tau)} \varphi_{1} \tan _{\tau}^{2}\left(\varphi_{2} \mu^{\tau}\right) \\
& \quad=2 \varphi_{1} \varphi_{2} \tan _{\tau}\left(\varphi_{2} \mu^{\tau}\right)\left(1+\tan _{\tau}^{2}\left(\varphi_{2} \mu^{\tau}\right)\right)
\end{aligned}
$$

and

$$
\begin{aligned}
& D^{(\tau)} \varphi_{1} \cot _{\tau}^{2}\left(\varphi_{2} \mu^{\tau}\right) \\
& \quad=-2 \varphi_{1} \varphi_{2} \cot _{\tau}\left(\varphi_{2} \mu^{\tau}\right)\left(1+\cot _{\tau}^{2}\left(\varphi_{2} \mu^{\tau}\right)\right),
\end{aligned}
$$

so that

$$
\begin{aligned}
& {\left[D^{(\tau)} \varphi_{1} \tan _{\tau}^{2}\left(\varphi_{2} \mu^{\tau}\right)\right]^{2}} \\
& \quad=\left(2 \varphi_{1} \varphi_{2}\right)^{2} \tan _{\tau}^{2}\left(\varphi_{2} \mu^{\tau}\right)\left(1+\tan _{\tau}^{2}\left(\varphi_{2} \mu^{\tau}\right)\right)^{2}
\end{aligned}
$$

and

$$
\begin{aligned}
& {\left[D^{(\tau)} \varphi_{1} \cot _{\tau}^{2}\left(\varphi_{2} \mu^{\tau}\right)\right]^{2}} \\
& \quad=\left(2 \varphi_{1} \varphi_{2}\right)^{2} \cot _{\tau}^{2}\left(\varphi_{2} \mu^{\tau}\right)\left(1+\cot _{\tau}^{2}\left(\varphi_{2} \mu^{\tau}\right)\right)^{2},
\end{aligned}
$$

which lead us to the following NLFODE:

$$
\begin{aligned}
& {\left[D^{(\tau)} \Phi_{\tau}(\mu)\right]^{2}} \\
& \quad=4 \varphi_{1} \varphi_{2}^{2} \Phi_{\tau}(\mu)\left(1+\frac{1}{\varphi_{1}} \Phi_{\tau}(\mu)\right)^{2} .
\end{aligned}
$$

Therefore, we get the following NLFODE:

$$
\begin{aligned}
& {\left[D^{(\tau)} \Phi_{\tau}(\mu)\right]^{2}} \\
& \quad=4 \varphi_{1} \varphi_{2}^{2} \Phi_{\tau}(\mu)\left(1+\frac{1}{\varphi_{1}} \Phi_{\tau}(\mu)\right)^{2},
\end{aligned}
$$

where the non-differentiable solutions are given by:

$$
\Phi_{\tau}(\mu)=\left\{\begin{array}{l}
\varphi_{1} \tan _{\tau}^{2}\left(\varphi_{2} \mu^{\tau}\right), \\
\varphi_{1} \cot _{\tau}^{2}\left(\varphi_{2} \mu^{\tau}\right) .
\end{array}\right.
$$

Let us suppose that the special functions defined on Canter sets can be expressed as follows:

$$
\Phi_{\tau}(\mu)=\varphi_{1} \tanh _{\tau}^{2}\left(\varphi_{1} \mu^{\tau}\right)
$$

and

$$
\Phi_{\tau}(\mu)=\varphi_{1} \operatorname{coth}_{\tau}^{2}\left(\varphi_{1} \mu^{\tau}\right) .
$$

Then, we have

$$
\begin{aligned}
& D^{(\tau)} \varphi_{1} \tanh _{\tau}^{2}\left(\varphi_{1} \mu^{\tau}\right) \\
& \quad=2 \varphi_{1} \varphi_{2} \tanh _{\tau}\left(\varphi_{1} \mu^{\tau}\right)\left(1+\tanh _{\tau}^{2}\left(\varphi_{2} \mu^{\tau}\right)\right) \\
& \quad D^{(\tau)} \varphi_{1} \operatorname{coth}_{\tau}^{2}\left(\varphi_{1} \mu^{\tau}\right) \\
& \quad=-2 \varphi_{1} \varphi_{2} \operatorname{coth}_{\tau}\left(\varphi_{1} \mu^{\tau}\right)\left(1+\operatorname{coth}_{\tau}^{2}\left(\varphi_{2} \mu^{\tau}\right)\right),
\end{aligned}
$$

and

so that

$$
\begin{aligned}
& {\left[D^{(\tau)} \varphi_{1} \tanh _{\tau}^{2}\left(\varphi_{1} \mu^{\tau}\right)\right]^{2}} \\
& \quad=\left(2 \varphi_{1} \varphi_{2}\right)^{2} \tanh _{\tau}^{2}\left(\varphi_{1} \mu^{\tau}\right)\left(1+\tanh _{\tau}^{2}\left(\varphi_{2} \mu^{\tau}\right)\right)^{2}
\end{aligned}
$$

and

$$
\begin{aligned}
& {\left[D^{(\tau)} \varphi_{1} \operatorname{coth}_{\tau}^{2}\left(\varphi_{1} \mu^{\tau}\right)\right]^{2}} \\
& \quad=\left(2 \varphi_{1} \varphi_{2}\right)^{2} \operatorname{coth}_{\tau}^{2}\left(\varphi_{1} \mu^{\tau}\right)\left(1+\operatorname{coth}_{\tau}^{2}\left(\varphi_{2} \mu^{\tau}\right)\right)^{2},
\end{aligned}
$$

which lead us to the following NLFODE:

$$
\begin{aligned}
& {\left[D^{(\tau)} \Phi_{\tau}(\mu)\right]^{2}} \\
& \quad=4 \varphi_{1} \varphi_{2}^{2} \Phi_{\tau}(\mu)\left(1+\frac{1}{\varphi_{1}} \Phi_{\tau}(\mu)\right)^{2} .
\end{aligned}
$$

In case the special functions defined on Cantor sets can be written as follows:

$$
\Phi_{\tau}(\mu)=\varphi_{1} \sec _{\tau}^{2}\left(\varphi_{2} \mu^{\tau}\right)
$$

and

$$
\Phi_{\tau}(\mu)=\varphi_{1} \csc _{\tau}^{2}\left(\varphi_{2} \mu^{\tau}\right)
$$

then we have

$$
\begin{aligned}
& D^{(\tau)} \varphi_{1} \sec _{\tau}^{2}\left(\varphi_{2} \mu^{\tau}\right) \\
& \quad=2 \varphi_{1} \varphi_{2} \sec _{\tau}^{2}\left(\varphi_{2} \mu^{\tau}\right) \tan _{\tau}\left(\varphi_{2} \mu^{\tau}\right)
\end{aligned}
$$

and

$$
\begin{aligned}
& D^{(\tau)} \varphi_{1} \csc _{\tau}^{2}\left(\varphi_{2} \mu^{\tau}\right) \\
& \quad=2 \varphi_{1} \varphi_{2} \csc _{\tau}^{2}\left(\varphi_{2} \mu^{\tau}\right) \cot _{\tau}\left(\varphi_{2} \mu^{\tau}\right) .
\end{aligned}
$$

Following Eqs. (96) and (97), we obtain

$$
\begin{aligned}
& {\left[D^{(\tau)} \varphi_{1} \sec _{\tau}^{2}\left(\varphi_{2} \mu^{\tau}\right)\right]^{2}} \\
& \quad=\left(2 \varphi_{1} \varphi_{2}\right)^{2} \sec _{\tau}^{4}\left(\varphi_{2} \mu^{\tau}\right)\left(\sec _{\tau}^{2}\left(\varphi_{2} \mu^{\tau}\right)-1\right)
\end{aligned}
$$

and

$$
\begin{aligned}
& {\left[D^{(\tau)} \varphi_{1} \csc _{\tau}^{2}\left(\varphi_{2} \mu^{\tau}\right)\right]^{2}} \\
& \quad=\left(2 \varphi_{1} \varphi_{2}\right)^{2} \csc _{\tau}^{4}\left(\varphi_{2} \mu^{\tau}\right)\left(\cot _{\tau}^{2}\left(\varphi_{2} \mu^{\tau}\right)-1\right),
\end{aligned}
$$

respectively. 
From Eqs. (98) and (99), we get the following NLFODE:

$$
\left[D^{(\tau)} \Phi_{\tau}(\mu)\right]^{2}=4 \varphi_{2}^{2} \Phi_{\tau}^{2}(\mu)\left(\frac{\Phi_{\tau}(\mu)}{\varphi_{1}}-1\right),
$$

where the non-differentiable solution is determined by

$$
\Phi_{\tau}(\mu)=\left\{\begin{array}{l}
\varphi_{1} \sec _{\tau}^{2}\left(\varphi_{2} \mu^{\tau}\right), \\
\varphi_{1} \csc _{\tau}^{2}\left(\varphi_{2} \mu^{\tau}\right) .
\end{array}\right.
$$

In the same manner, we establish the following special function defined on Cantor sets (see Ref. 40):

$$
\Phi_{\tau}(\mu)=\varphi_{1} \sec h_{\tau}^{2}\left(\varphi_{2} \mu^{\tau}\right),
$$

which leads to

$$
\begin{aligned}
& D^{(\tau)} \varphi_{1} \sec h_{\tau}^{2}\left(\varphi_{2} \mu^{\tau}\right) \\
& \quad=-2 \varphi_{1} \varphi_{2} \sec h_{\tau}^{2}\left(\varphi_{2} \mu^{\tau}\right) \tan h_{\tau}\left(\varphi_{2} \mu^{\tau}\right) .
\end{aligned}
$$

In view of Eq. (103), we have the following NLFODE (see Ref. 40):

$$
\left[D^{(\tau)} \Phi_{\tau}(\mu)\right]^{2}=4 \varphi_{2}^{2} \Phi_{\tau}^{2}(\mu)\left(1-\frac{\Phi_{\tau}(\mu)}{\varphi_{1}}\right) .
$$

Equation (104) was used to find the traveling-wave solution for the fractal Korteweg-de Vries equation within LFD (see, for details, Ref. 40).

\section{CONCLUSION}

In our present work, the fractal special functions defined on Cantor sets were structured for the first time. With the use of the LFDs of the given special functions, we proposed the NLFODEs and their exact solutions of non-differentiable type. The results are applicable for designing the exact traveling-wave solutions for the nonlinear FPDEs in mathematical physics (see Ref. 40).

\section{ACKNOWLEDGMENTS}

This work is supported by the State Key Research Development Program of the People's Republic of China (Grant No. 2016YFC0600705), the Natural Science Foundation of China (Grant No. 51323004), and the Priority Academic Program Development of Jiangsu Higher Education Institutions (PAPD2014).

\section{REFERENCES}

1. A. A. Kilbas, H. M. Srivastava and J. J. Trujillo, Theory and Applications of Fractional Differential Equations (Elsevier Science Publishers, UK, 2006).
2. F. Mainardi, Fractional Calculus and Waves in Linear Viscoelasticity: An Introduction to Mathematical Models (World Scientific Publishing Company, Singapore, 2010).

3. J. A. T. Machado, F. Mainardi and V. Kiryakova, Fractional calculus: Quo vadimus? (Where are we going?), Fract. Calc. Appl. Anal. 18 (2015) 495526.

4. F. Mainardi and R. Gorenflo, On Mittag-Lefflertype functions in fractional evolution processes, $J$. Comput. Appl. Math. 118 (2000) 283-299.

5. Y. Li, Y. Chen and I. Podlubny, Mittag-Leffler stability of fractional order nonlinear dynamic systems, Automatica 45 (2009) 1965-1969.

6. M. D. Ortigueira, An introduction to the fractional continuous-time linear systems: The 21st century systems, IEEE Circuits Syst. Mag. 8(3) (2008) $19-26$.

7. A. G. Radwan, A. S. Elwakil and A. M. Soliman, Fractional-order sinusoidal oscillators: Design procedure and practical examples, IEEE Trans. Circuits. Syst. 55 (2008) 2051-2063.

8. A. S. Elwakil, Fractional-order circuits and systems: An emerging interdisciplinary research area, IEEE Circuits Syst. Mag. 10(4) (2010) 40-50.

9. I. Petras, Fractional-order memristor-based Chua's circuit, IEEE Trans. Circuits Syst. 57(12) (2010) 975-979.

10. H. M. Srivastava, Some families of Mittag-Leffler type functions and associated operators of fractional calculus, TWMS J. Pure Appl. Math. 7 (2016), 123-145.

11. F. Mainardi, Fractional relaxation-oscillation and fractional diffusion-wave phenomena, Chaos Solitons Fractals 7 (1996) 1461-1477.

12. R. Gorenflo, F. Mainardi and H. M. Srivastava, Special functions in fractional relaxation-oscillation and fractional diffusion-wave phenomena, in Proceedings of the Eighth International Colloquium on Differential Equations, D. Bainov (Ed.) (Plovdiv, Bulgaria, 18-23 August 1997) (VSP Publishers, Utrecht and Tokyo, 1998), pp. 195-202.

13. H. Schiessel and A. Blumen, Hierarchical analogues to fractional relaxation equations, J. Phys. A, Math. Gen. 26(19) (1993) 5057.

14. R. Metzler and T. F. Nonnenmacher, Fractional relaxation processes and fractional rheological models for the description of a class of viscoelastic materials, Int. J. Plasticity 19 (2003) 941-959.

15. Y. Ding and H. Ye, A fractional-order differential equation model of HIV infection of CD4+ T-cells, Math. Comput. Model. 50 (2009) 386-392.

16. R. P. Agarwal, V. Lakshmikantham and J. J. Nieto, On the concept of solution for fractional differential equations with uncertainty, Nonlinear Anal. 72 (2010) 2859-2862. 
17. V. Daftardar-Gejji and H. Jafari, Adomian decomposition: A tool for solving a system of fractional differential equations, J. Math. Anal. Appl. 301 (2005) $508-518$.

18. J. S. Duan, T. Chaolu and R. Rach, Solutions of the initial value problem for nonlinear fractional ordinary differential equations by the RachAdomian-Meyers modified decomposition method, Appl. Math. Comput. 218 (2012) 8370-8392.

19. M. Zayernouri and G. E. Karniadakis, Exponentially accurate spectral and spectral element methods for fractional ODEs, J. Comput. Phys. 257 (2014) 460480.

20. C. Li and F. Zeng, The finite difference methods for fractional ordinary differential equations, Numer. Funct. Anal. Optim. 34(2) (2013) 149-179.

21. R. Lin and F. Liu, Fractional high order methods for the nonlinear fractional ordinary differential equation, Nonlinear Anal. 66 (2007) 856-869.

22. V. S. Ertürk and S. Momani, Solving systems of fractional differential equations using differential transform method, J. Comput. Appl. Math. 215 (2008) $142-151$.

23. Y. F. Luchko and H. M. Srivastava, The exact solution of certain differential equations of fractional order by using operational calculus, Comput. Math. Appl. 29(8) (1995) 73-85.

24. C. Cattani, H. M. Srivastava and X.-J. Yang, Fractional Dynamics (De Gruyter, Warsaw, Poland, 2015).

25. B. West, M. Bologna and P. Grigolini, Physics of Fractal Operators (Springer, Berlin, 2012).

26. C. Cattani and A. Ciancio, On the fractal distribution of primes and prime-indexed primes by the binary image analysis, Physica. A 460 (2016) 222229.

27. V. E. Tarasov, Electromagnetic waves in non-integer dimensional spaces and fractals, Chaos Solitons Fractals 81 (2015) 38-42.

28. V. E. Tarasov, Fractal electrodynamics via noninteger dimensional space approach, Phys. Lett. A 379 (2015) 2055-2061.

29. A. S. Balankin, B. Mena, O. Susarrey and D. Samayoa, Steady laminar flow of fractal fluids, Phys. Lett. A 381 (2017) 623-628.

30. A. Carpinteri, P. Cornetti and A. Sapora, Statickinematic fractional operators for fractal and nonlocal solids, ZAMM J, Appl. Math. Mech. 89 (2009) 207-217.

31. A. Carpinteri, B. Chiaia and P. Cornetti, A fractal theory for the mechanics of elastic materials, Mater. Sci. Eng. A 365(1) (2004) 235-240.

32. M. Ostoja-Starzewski, Towards thermoelasticity of fractal media, J. Therm. Stresses 30 (2007) 889-896.

33. A. M. Lopes, J. A. T. Machado, Modeling vegetable fractals by means of fractional-order equations, $J$. Vib. Control 22 (2016) 2100-2108.
34. S. Liu, Z. Pan, W. Fu and X. Cheng, Fractal generation method based on asymptote family of generalized Mandelbrot set and its application, J. Nonlinear Sci. Appl. 10(3) (2017) 1148-1161.

35. S. Liu, W. Fu, L. He, J. Zhou and M. Ma, Distribution of primary additional errors in fractal encoding method, Multimed. Tools Appl. 76(4) (2017) 57875802 .

36. W. Fu, J. Zhou, S. Liu, M. Ma and Y. Ma, Differential trajectory tracking with automatic learning of background reconstruction, Multimed. Tools Appl. 75(21) (2016) 13001-13013.

37. S. Liu, Z. Zhang, L. Qi and M. Ma, A fractal image encoding method based on statistical loss used in agricultural image compression, Multimed. Tools Appl. 75(23) (2016) 15525-15536.

38. X.-J. Yang, J. A. T. Machado, C. Cattani and F. Gao, On a fractal LC-electric circuit modeled by local fractional calculus, Common. Nonlinear Sci. Numer. Simul. 47 (2017) 200-206.

39. X.-J. Yang and H. M. Srivastava, An asymptotic perturbation solution for a linear oscillator of free damped vibrations in fractal medium described by local fractional derivatives, Common. Nonlinear Sci. Numer. Simul. 29 (2015) 499-504.

40. X.-J. Yang, J. A. T. Machado, D. Baleanu and C. Cattani, On exact traveling-wave solutions for local fractional Korteweg-de Vries equation, Chaos 26(8) (2016) Article ID 084312.

41. X.-J. Yang and J. A. T. Machado, A new insight into complexity from the local fractional calculus view point: Modelling growths of populations, Methods Meth. Appl. Sci., doi: 10.1002/mma.3765.

42. X.-J. Yang, J. A. T. Machado and J. J. Nieto, A new family of the local fractional PDEs, Fundam. Inf. 151(1-4) (2017) 63-75.

43. X.-J. Yang, D. Baleanu and H. M. Srivastava, Local Fractional Integral Transforms and Their Applications (Academic Press, London, 2015).

44. D. Kumar, J. Singh and D. Baleanu, A hybrid computational approach for Klein-Gordon equations on Cantor sets, Nonlinear Dyn. 87 (2017) 511-517.

45. D. Baleanu, H. Khan, H. Jafari and R. A. Khan, On the exact solution of wave equations on cantor sets, Entropy 17 (2015) 6229-6237.

46. Y. Pandir and H. H. Duzgun, New exact solutions of time fractional Gardner equation by using new version of F-expansion method, Common. Theor. Phys. 67(1) (2017) 1-9.

47. S. S. Ray and S. Sahoo, New exact solutions of time fractional modified Kawahara equations in modelling surface tension in shallow-water and capillary gravity water waves, Eur. Phys. J. Plus 132(1) (2017) 1-9.

48. M. Kaplan and A. Bekir, Construction of exact solutions to the space-time fractional differential equations via new approach, Optik 132 (2017) 1-8. 